\title{
Robotics in Interventional Radiology: Past, Present, and Future
}

\begin{abstract}
Robotic endovascular systems have been successfully used to treat a wide range of pathologies including endovascular aneurysm repairs, uterine artery embolizations, and cardiac electrophysiology ablations. Limited research suggests that the use of robotic systems may help to achieve a more accurate manipulation and increased steerability of the catheter during endovascular procedures. In addition, robotic systems have also been designed to enhance image-guided percutaneous procedures, demonstrating a potential to facilitate needle placements and guidance and diminish radiation exposure risk. There are still many limitations for the widespread of this emerging technology. More studies are needed to validate the use of robotic systems and to show meaningful clinical advantages over traditional methods as well as assessing cost-effectiveness.
\end{abstract}

Keywords: Image-guided robotic systems, interventional radiology, robotic endovascular systems, robotics

\section{Introduction}

Interventional radiology (IR) is a relatively new field with $<100$ years since its foundations. Although there is a previous documentation of interventional procedures, IR is born on the basis of Charles Dotter work in 1963. ${ }^{[1]}$ His pioneer labor in angioplasty and vascular radiology sets ground for the creation of a new medical specialty, IR. ${ }^{[2]}$ Since then, IR has grown rapidly and now covers a broad spectrum of procedures. An escalating role of IR has been noticeable by the increased complexity of the procedures and the enlarged range of conditions it now covers.

In contrast to open surgery, IR depends on minimally invasive approaches and therefore relies on multimodal radiological image guidance and patient anatomy for precise targeting and therapy. ${ }^{[3]}$ Improved imaging of internal structures with better and safer contrast agents has given IR a protagonist role and more demand on percutaneous peripheral vascular interventions ${ }^{[4]}$ Compared to more invasive surgeries, IR procedures have shown to be safe and cost-effective with less complication rates, duration of hospital stays, and less recovery periods. ${ }^{[5]}$

The adoption of novel techniques is particularly inherent to IR. Medical

This is an open access journal, and articles are distributed under the terms of the Creative Commons AttributionNonCommercial-ShareAlike 4.0 License, which allows others to remix, tweak, and build upon the work non-commercially, as long as appropriate credit is given and the new creations are licensed under the identical terms.

For reprints contact: reprints@medknow.com robotics has produced a paradigm shift in therapy of various pathologies since the introduction of the Vinci system, the first robotic system approved by the Food and Drug Administration (FDA) in 2000, now used in general laparoscopic surgeries and minimally invasive prostatectomies. ${ }^{[6]}$ The greatest impact of robotic surgery is the increased precision and accuracy during tissue manipulation. ${ }^{[7]}$ This principle inspired research to extend the use of robotic assistance for image-guided percutaneous interventions and endovascular procedures. This paper provides an overview of the use and impact of robots in multiple domains of IR as well as a brief outline on the latest mayor technological innovations in the field.

\section{Steerable Robotic Catheter Systems}

Hansen Medical (Mountain View, California) developed two generations of robotic endovascular systems. The first one, the Sensei $\mathrm{X}$ robotic catheter system, is a robot with FDA approval since 2007 designed to achieve more accurate manipulation and increased steerability of the catheter during cardiovascular procedures. The Artisan Extend Control Catheter is a robotically steerable sheath manufactured to work with this system. The catheter consists of a flexible, multidirectional inner guide 11-F outer

How to cite this article: Rueda MA, Riga CT, Hamady MS. Robotics in interventional radiology: Past, present, and future. Arab J Intervent Radiol 2018;2:56-63.

\section{Maria Alejandra \\ Rueda ${ }^{1}$, Celia Theodoreli Riga $^{2}$, Mohamad S. Hamady ${ }^{1,2}$}

${ }^{I}$ Division of Interventional Radiology, Imperial College NHS Trust, ${ }^{2}$ Department of Surgery and Cancer, Imperial College, London, UK

\section{Address for correspondence: Dr. Maria Alejandra Rueda, Division of Interventional Radiology, Imperial College NHS Trust, London, UK. E-mail: mariarueda91@gmail. com}

\section{Access this article online}

Website: www.arabjir.com

DOI: 10.4103/AJIR.AJIR_25_18

Quick Response Code:

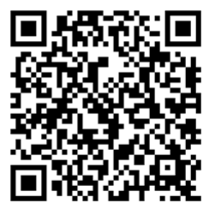


diameter and 8.5-F inner diameter, within a unidirectional outer guide sheath $14-\mathrm{F}$ outer diameter and 11-F inner diameter. ${ }^{[8]}$ They have been used in numerous endovascular procedures. Initially, its main used was focused on cardiac electrophysiology ablation. Dello Russo et al. used the Sensei $\mathrm{X}^{\mathrm{TM}}$ robotic navigation system and the Artisan Extend Catheter to increase contact force during atrial fibrillation ablation with good results. ${ }^{[9]}$ Other applications have been explored for this robotic system and catheter. Technical feasibility has been demonstrated in other procedures such as endovascular aneurysm repair (EVAR), treatment of pulmonary stenosis following single-lung transplant, and stenting of right renal branch during fenestrated stent graft. ${ }^{[10-12]}$

The next generation system developed by Hansen Medical is called The Magellan robotic system. Unlike the previous system, The Magellan robotic system was designed specifically to navigate peripheral arteries. It consists of a workstation with a control screen and the controller device where the physician has access to parameters such as torque position, catheter angulation, and rotational position. ${ }^{[13]}$ It also counts with an electromagnetic catheter manipulator and a number of steerable guide catheters. The system counts with three FDA-approved specialized catheters. They range from 50 to $102 \mathrm{~cm}$ in length and have outer diameters of 10,9 , and 6 Fr. ${ }^{[14,15]}$

Stent grafting of aneurysms through endovascular approach is becoming a more frequent procedure for interventional radiologists. In the presence of challenging anatomy with tortuous iliac arteries, atherosclerosis and angulated visceral aortic segment technical difficulties may arise making the procedure more time-consuming. Available catheters have premade shapes and intrinsic curve design that limit maneuverability, tip control, and target access. ${ }^{[16]}$ Imperial College Endovascular Team have adapted the use of the Magellan robotic catheter steering system in phantom and porcine models. ${ }^{[17,18]}$ Later, they published a case report of a robot-assisted fenestrated EVAR procedure, demonstrating its safety and achievability. ${ }^{[19]}$ All of these works showed reduction in vessel cannulation times and instrumentation, precise positioning, and significant reduction of radiation exposure when using the robotic systems. At the moment, there are no randomized clinical trials that can corroborate any further these early findings. ${ }^{[20]}$

The Hansen Medical Vascular Catheter Control System has been successfully used to treat a wide range of other pathologies. For example, Bismuth et al. used it to successfully treat iliac and femoral artery lesions. Interestingly, they also found that although the system could be used by all interventionalists regardless of their experience, a statistically significant difference in navigation time and total fluoroscopy times between experienced and unexperienced interventionalists was observed. ${ }^{[21]}$ Figures 1 and 2 show an example of an embolization of left internal iliac artery aneurysm. Rolls et al. published the first in woman safety evaluation of the Magellan system with the NorthStar catheter. They successfully performed bilateral uterine artery embolization in five women noticing the robotic system may facilitate catheterization of small iliac artery divisions specially in complex anatomy. ${ }^{[14]}$ Finally, Bagla et al. performed 40 prostatic artery embolizations, showing that a wider range of pelvic pathologies in both men and woman can be addressed successfully and safely with robotic systems, Figure 3 shows an example of a successful robotic-assisted prostate artery embolization.

There are other commercially available remote catheter navigation systems with electromechanical technology intended for percutaneous coronary interventions and radiofrequency $(\mathrm{RF})$ ablations. The Amigo remote catheter system (Catheter Precision Inc., NJ, USA) is a robotic arm with three degrees of freedom (DOF) that attaches to the electrophysiology table. Amigo is designed to adapt many commercially available catheters that are manipulated remotely, up to $30 \mathrm{~m}$ away from the patient. Catheters are visualized using conventional fluoroscopic techniques. The operator can produce three types of catheter movements while monitoring using standard fluoroscopic techniques: deflection, rotation, and linear motion. Khan et al. published a nonrandomized prospective clinical trial validating the safe and effective use of the Amigo remote catheter system for positioning a mapping catheter within the right atrium and ventricle. ${ }^{[22]}$

The CorPath 200 and its second generation CorPath GRX (Corindus Vascular Robotics, MA, USA) are intended to assist in percutaneous coronary interventions. This complex robot is composed of a control console for the physician to control the catheter remotely and a cassette mounted on a robotic drive in the robotic arm at bedside. ${ }^{[23]}$ Weisz et al. published a pivot multicenter study where 164 patients underwent percutaneous intervention with the CorPath 200. The study showed technical success of $98.8 \%$ and clinical success of $97.6 \%$. In addition, radiation exposure to the operator was reduced in $95.2 \%$ compared to traditional methods. ${ }^{[24]}$ The CathROB is also an electromechanical robotic device made to manipulate steerable electrophysiology catheters. It has a compact and lightweight design comprising a motion unit, a central unit, and a remote user interface. The system has been used by Cercenelli et al. in in vitro and in vivo animal evaluations with good accuracy and adequate tissue contact. ${ }^{[25]}$

The Niobe remote magnetic navigation system and the Vdrive robotic navigation system (Stereotaxis Inc., MO, USA) are electromagnetically driven systems used to navigate especially designed catheters. Two external magnets are positioned on each side of the patient allowing movement of the catheter within three DOF. Due to these external magnets, the system is inherently incompatible with real-time use of magnetic resonance imaging (MRI) 
in procedure guidance. ${ }^{[26]}$ A newer version the Niobe ES was used for catheter ablation of atrial fibrillation reducing operating and fluoroscopy times. ${ }^{[27]}$

All of these studies suggest that robotic catheterization can provide superior maneuverability compared to traditional techniques, especially in angulated vessel segments and in unfavorable artery access. In addition, robotic systems such as the Magellan robot could avoid complications such as dissection or rupture and may expand the pathologies and patients suitable for endovascular treatment. ${ }^{[28]}$ Finally, one of the biggest benefits of the robotic-assisted systems is the ability to perform safer procedures outside the radiation zone. Rao published seven cases of transarterial chemoembolization using the Magellan robot, showing an average reduction of over $80 \%$ in radiation exposure

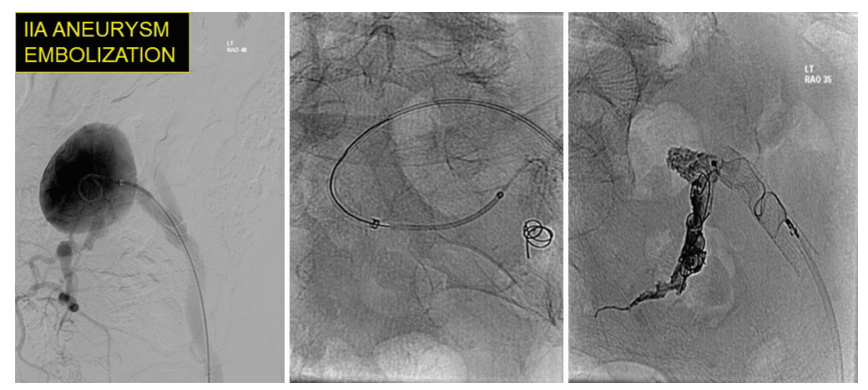

Figure 1: Embolization of left internal iliac artery aneurysm using Onyx and occlusion Amplatzer device

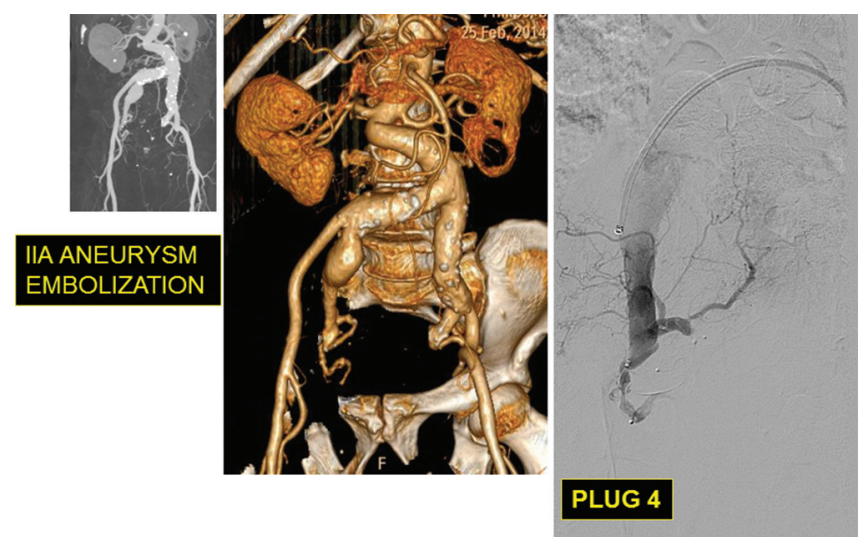

Figure 2: Embolization of right internal iliac artery using Amplatzer occlusion device to the operator. ${ }^{[29]}$ Definite downsides of these systems that have not allowed their commercial availability are the increased cost with this technology. Fitting Riga et al. calculations, $\$ 600,000$, are needed as an initial capital to acquire Magellan robotic system. In addition, maintenance costs are around $\$ 80,000$, and each disposable robotic catheter can cost a minimum of $\$ 1500$ per catheter. ${ }^{[30]}$ This was perhaps one of the main reasons that prohibited wide adoption in the clinical community and led eventually to discontinue production of those particular robot type. The newer systems, such as CorPath GRX (Corindus Vascular Robotics, MA, USA), is yet to be evaluated both clinically and commercially. At present, there are no large cohort studies, randomized trials, or cost-effectiveness analysis that can encourage to greatly adopt this technology.

\section{Computed Tomography-Guided Robotic Systems}

Image-guided percutaneous puncturing techniques entail meticulous path planning and navigation. Adequate visualization of target by means of different image-guiding modalities such as fluoroscopy, ultrasound (US), computed tomography (CT), and MRI is crucial for the success of the intervention. ${ }^{[31]}$ In clinical practice, the interventional radiologist has to assess inherent advantages and disadvantages of each modality for correct selection and application and even consider multi-image techniques.

Percutaneous CT-guided needle interventions are common in medical practice given that they are minimally invasive procedures that can be very effective for image-guided biopsies and for therapies such as tumor ablations and infiltrations. ${ }^{[32]}$ CT-guided procedures have great benefits in terms of costs, availability, and visibility of anatomical structures specially when visualizing deep tissues. ${ }^{[32,33]}$ The success of CT and CT fluoroscopy-guided biopsies varies from $90 \%$ to $100 \%$ and $83 \%$ to $100 \%$, respectively. ${ }^{[34]}$ Diagnostic yield and procedure outcome is dependent upon physicians' experience but also upon lesion site and anatomy. When the location of the target prompts a risk of damage to neighbor structures, there is low tolerance in needle placement errors. In cases where there is limited entry site and when access is restricted due to angulations and anatomy, an increase in patients
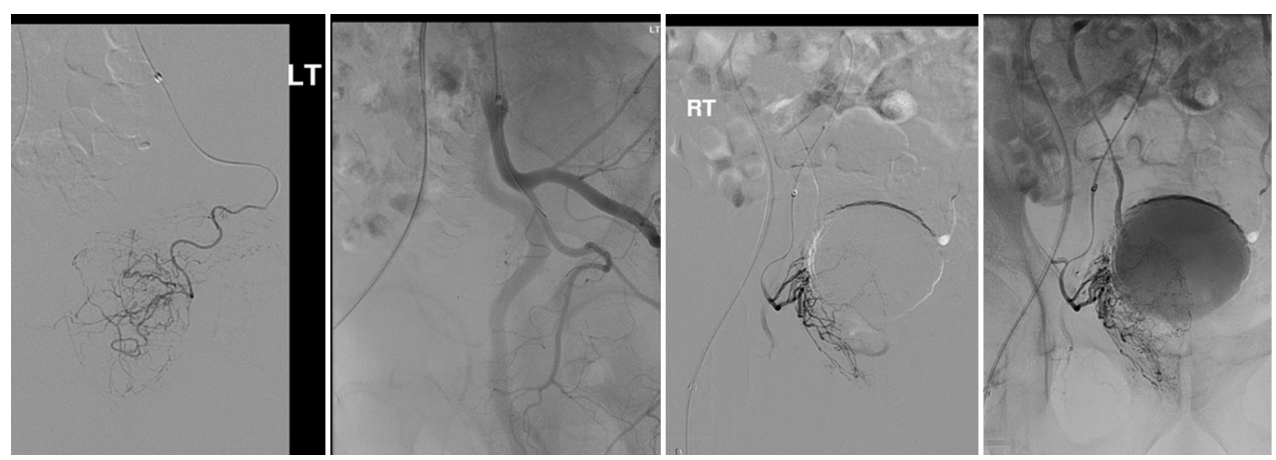

Figure 3: Robotic cannulation and embolization of bilateral prostatic arteries 
and physicians' radiation exposure is a risk due to longer procedure times because of needle adjustments. ${ }^{[32]}$ CT fluoroscopy has allowed for shorter procedure times and more success rates compared to conventional CT. In both of their studies, Gianfelice et al. and Silverman et al. showed statistically significant decreased needle placement times using CT fluoroscopy. ${ }^{[35,36]}$ The setback is that CT fluoroscopy implies much higher radiation both to the patient and to the physician. Kato et al. calculated that a physician with continuous hand exposure could only perform four CT fluoroscopic procedures each year on the basis of an annual dose limit of $500 \mathrm{mSv}^{[37]}$ Although there are a number of techniques to minimize radiation exposure, robotic assistance offers to address this matters by facilitating needle placements and guidance and diminishing the radiation exposure risk.

There have been several robotic systems designed to work with CT. The AcuBot robot was one of the first, developed in the URobotics Laboratory at Johns Hopkins Medical Institutions (Baltimore, USA). The AcuBot has six DOF and it includes three previous robotic modules developed by the company: (1) the percutaneous access to the kidney (PAKY) radiolucent needle driver where the instrument would be loaded, (2) a remote center or motion that holds PAKY and is capable of needle orientation, and (3) a passive position S-arm mounted on a bridge frame. In addition, the robot is composed of (4) An XYZ Cartesian stage for positioning and (5) a user interface with controls and a two-axis joystick. ${ }^{[38,39]}$

This robotic system was tested in a cadaveric study by Stoianovici et al. proving its feasibility in perispinal and facet blocks. ${ }^{[38,40]}$ The PAKY and the RCM (Remote Center of Motion) robot was tested in a phantom study showing that the angular error between the ideal trajectory to target and the actual robot's trajectory was $0.61^{\circ}$. Subsequently, they successfully used the robot in numerous clinical cases including several core needle biopsies, radiofrequency ablations (RF), nephrostomy tube placements and neobladder access. Although no statistical data was generated, the value of this studies is, they showed radiation exposure to the physician could be reduced and in fact it can be completely avoided. Moreover, radiation to the patient can also be diminished by avoiding continuous imaging when advancing the needle given the reliability of the robot when respiration is controlled. There is still much research to be done; the robot showed mayor limitations such as extra preparation time, issues with the RF probes, and absence of tactile feel and force feedback when handling the joystick. ${ }^{[41,42]}$

The B-Rob I is another robotic system developed by Austrian Research Center (ARC) - Seibersdorf, Austria. together with the Departments of Diagnostic Radiology and Biomedical Engineering and Physics, Vienna Medical University. The system was developed, so it can work with both CT scanners and US systems. This seven DOF robot is integrated to a mobile rack with a four DOF needle positioning system in a three Cartesian plane plus a rotational link component for needle orientation. The system includes an optical tracker system (Polaris, Northern Digital, Bakersfield, CA, USA) as well as the planning and monitoring software. ${ }^{[39]}$ The robot uses active joints to position the instrument, but the insertion of the needle has to be performed manually by the physician. This system was used in several phantom studies ${ }^{[43,44]}$ showing its feasibility. Nonetheless, given it was difficult to integrate in the clinic and it was not practical, a second prototype B-Rob II was designed. Martinez et al. used this latest prototype in a phantom model performing 21 biopsies. He attained an average needle placement accuracy of $1.8 \mathrm{~mm}( \pm 1.1 \mathrm{~mm})$ using robotic assistance with average of $2 \mathrm{~min} 21 \mathrm{~s}$ per procedure. ${ }^{[45]}$

Maurin et al. presented in 2008 a novel robotic system called the CT-Bot. It consists of a patient-mounted parallel structure with three DOF for position, two DOF for orientation, and an extra DOF for needle self-rotation. It has an accuracy of $5-\mathrm{mm}$ position error at the tip of a 200-mm long needle. ${ }^{[46]}$ The system counts with haptic interface and ultrasonic motors that allow the needle to be held firmly throughout the procedure. Although some experiments have been conducted to show its feasibility and accuracy at object targeting, this prototype is not available commercially and had not been explored further.

The Robopsy system, introduced by Walsh et al. is a patient-mounted, telerobotic needle guidance and insertion system for more accurate targeting during CT-guided biopsies. Robopsy was originally designed to perform percutaneous lung biopsies. The needle is intended to be guided by the interventionist remotely while allowing simultaneous imaging. The robot counts with four DOF, two for needle orientation, one for needle gripping, and one for needle insertion and retraction. ${ }^{[47]}$ Furthermore, Seitel et al. developed an interface for the Robopsy that is directly integrated with the medical imaging data to provide specificity in trajectory and automatically calculate insertion parameters. ${ }^{[48]}$

There have been other robotic systems developed, for example, the robotic IR assistance platform (MAXIO; Perfint Healthcare, Chennai, India) described by Koethe et al. in 2014. ${ }^{[32]}$ The iSYS Medizintechnik and its newer version the iSYS1 have been tested in phantom studies with good results. ${ }^{[49]}$ Perfint Healthcare Pvt. Ltd. holds two commercially available robotic systems. The ROBIO EX which is a CT and positron emission tomography-CT robotic positioning systems for assisted thoracoabdominal interventions and the ROBIO EZ a robotic system with 5 DOF used for needle placement during CT procedures. ${ }^{[50]}$ Zhou et al. used a Mitsubishi RV-E2 6 DOF (developed in the Stony Brook University, NY, USA) articulated robot 
with a respiratory phase tracking to propose a robotic needle placement technique for accurately biopsying lung nodules during respiratory motion. ${ }^{[1]}$ Finally, the Institute of Robotics and Mechatronics at the German Aerospace Center in collaboration with KUKA roboter setup a third generation of torque-controlled robot called the Light Weight Robot III. It is a seven DOF robot counting with a tracking system for needle guide and registering with an angiographic $\mathrm{C}$-arm. ${ }^{[52]}$

\section{Magnetic Resonance Imaging-Guided Robotic Systems}

INNOMOTION (Innomedic, Herxheim and FZK Karlsruhe Germany and TH Gelsenkir) is a pneumatic robotic assistance platform consisting of a six DOF robot arm attached to an arch that is mounted to the patient table. The arm can be positioned according to the area of interest within the body. ${ }^{[39,53]}$ The INNOMOTION has been assessed in the clinic by Zangos et al. when they used this robotic system and a 1.5-T MR system to perform image-guided biopsies using a transgluteal approach in patients with suspicious prostate lesions. ${ }^{[54]}$ Kettenbach et al. also evaluated its feasibility when performing percutaneous biopsy, drainage, and tumor ablation within the chest, abdomen, and retroperitoneum. ${ }^{[55]}$ Since the INNOMOTION is also compatible with CT, it has been compared with other systems, finding that it is less flexible in selecting entry points compared to other robotic systems such as AcuBot or iSYS1 system. In addition, a mayor disadvantage is that it is very large, and especially for breast interventions, it is difficult to use with breast coils because of the patient's position. Yang et al. developed a six DOF slave robotic platform and a one DOF needle driver designed for MRI-guided breast biopsies. ${ }^{[56]}$ Park et al. also described an image-guided intervention robot system designed for use with the General Electric (GE) Signa HDxt 3.0 Tesla MRI system (GE Healthcare, Waukesha, Wisconsin, USA) for breast cancer patients that overcomes the space limitations in the MRI gantry. ${ }^{[57]}$

MrBot is a six DOF robot for image-guided transperineal prostate percutaneous access. It is compatible with all MRI scanners, even the ones with the highest field strength. It is made up of electrically nonconductive and nonmagnetic materials such as plastics, ceramics, and rubbers and has especially designed motor with all electric components outside the imager room. ${ }^{[58]}$ MrBot has FDA approval for a biopsy trial that has been successfully performed in five patients with a needle target accuracy of $2.55 \mathrm{~mm} .^{[59]}$ Another robot for percutaneous interventions is The Light Puncture Robot (LPR) being developed in Grenoble. This robotic system has 5 DOF, dimensions of $15 \times 23 \mathrm{~cm}$ and only weights $1 \mathrm{~kg}$. It is compatible with both CT and MRI; therefore, it is made out of plastic and uses pneumatic actuators powered by compressed air. ${ }^{[60]}$ Initial phantom and animal experiments have been conducted with this robotic device apparently with good reproducibility. Nonetheless, translation accuracy of the robot over the patient's body surface is only satisfactory over distances $<30 \mathrm{~mm}$, having an inaccurate displacement above this limit. ${ }^{[61]}$

\section{Ultrasound-Guided Robotic Systems}

US is an inexpensive widely available technique with frequent use in clinical diagnosis and intervention. Due to the extreme operator-dependent nature of US imaging, robotic application and research in this area have been slow. Nonetheless, it is precisely because of the need of a skilled sonographer that robotic-assisted US could aid to standardize its use and make it more widely available in remote rural areas. Autonomous US imaging systems need a tracking system to measure the position of the probe. This information can be obtained through optical tracking, electromagnetic tracking, and passive encoded mechanical systems. ${ }^{[62]}$

Boctor et al. used an LBM/JHU LARS 6 DOF dual-arm robot with electromagnetic tracking system equipped with torque and force sensor. This autonomous US imaging robot was tested in animal phantom to perform needle placements with a 3-mm error reported; nonetheless, no further work has been performed. ${ }^{[63]}$ Liang et al. proposed a real-time three-dimensional (3D) US-guided multiple core biopsy to be autonomously conducted by a $6+1$ DOF robotic arm with a success rate of $93 \%$ in a phantom study with turkey breasts. ${ }^{[64]}$ Other autonomous robotic US imaging systems are being developed like the one by Mebarki et al., a six DOF robot intended to guide 2D US probe accurately with respect to an observed object. ${ }^{[65]}$ Janvier et al. ${ }^{[66]}$ developed a robot prototype to provide accurate $3 \mathrm{D}$ US scanning of lower limb vessels.

Teleoperated US imaging has been developed to allow a skilled radiologist to remotely operate the device. The radiologist at the master site can control through a joystick or a haptic device the robot at the slave site. ${ }^{[62]}$ Real-time US image is provided for the radiologist to use for diagnostic purposes. Many ongoing projects have encouraged the development of these devices, for example, the Advanced Robotised Tele-sonography Integrated Service project by the European Space Agency. ${ }^{[62,67]}$ Other similar projects include the TERESA project to bring astronaut quality US examinations $^{[68]}$ and the ESTELE ${ }^{[69]}$ and PROSIT ${ }^{[70]}$ projects for abdominal and fetal echography.

\section{Conclusions}

Although initial case studies and case reports have shown that robotic assistance has superior maneuverability in endovascular procedures, it is important to acknowledge that it will not eliminate the need for a learning curve and for an experienced interventional radiologist. Great 
advances in imaging techniques and its fusion with robotic systems seem to have great promise for image-guided percutaneous procedures specially to reduce radiation exposure risk. Main limitations include lack of haptic feedback, robotic catheter compatibility with various stents and balloons, lack of smooth workflow within the angiography suite setting, and high capital and/ or consumable cost. Technological refinement led by interventional radiologists in collaboration with industry, and more studies are needed to advance technological maturity and to show meaningful clinical advantages over traditional methods as well as assessing cost-effectiveness.

\section{Acknowledgement}

This work is funded by grants and infrastructure support provided by the NIHR Imperial Biomedical Research Centre (BRC).

\section{Financial support and sponsorship}

Nil.

\section{Conflicts of interest}

There are no conflicts of interest.

\section{References}

1. Tang Z, Jia A, Li L, Li C. Brief history of interventional radiology. Zhonghua Yi Shi Za Zhi 2014;44:158-65.

2. Payne MM. Charles theodore dotter: The father of intervention. Texas Heart Inst J 2001;28:28-38.

3. Mewes A, Hensen B, Wacker F, Hansen C. Touchless interaction with software in interventional radiology and surgery: A systematic literature review. Int J Comput Assist Radiol Surg 2017;12:291-305.

4. ESR Executive Council 2009 and European Society of Radiology. The professional and organizational future of imaging. Insights Into Imaging (Berlin/Heidelberg) 2010;1:12-20.

5. Di Marco L, Anderson MB. The new interventional radiology/ diagnostic radiology dual certificate: "Higher standards, better education." Insights Into Imaging (Berlin Heidelberg) 2016;7:163-5.

6. Samadi BD. Robotic Oncology, History and the future of Robotic Surgery. Available from: http://www.roboticoncology. com/history-of-robotic-surgery/. [Last accessed on 2018 Jan 05].

7. Beasley RA. Medical Robots: Current Systems and Research Directions. J Robot. 2012;2012:1-14.

8. Antoniou GA, Riga CV, Mayer EK, Cheshire NJ, Bicknell CD. Clinical applications of robotic technology in vascular and endovascular surgery. J Vasc Surg 2011;53:493-9.

9. Dello Russo A, Fassini G, Conti S, Casella M, Di Monaco A, Russo E, et al. Analysis of catheter contact force during atrial fibrillation ablation using the robotic navigation system: Results from a randomized study. J Interv Card Electrophysiol 2016;46:97-103.

10. Lumsden AB, Anaya-Ayala JE, Birnbaum I, Davies MG, Bismuth J, Cheema ZF, et al. Robot-assisted stenting of a high-grade anastomotic pulmonary artery stenosis following single lung transplantation. J Endovasc Ther 2010;17:612-6.

11. Carrell T, Dastur N, Salter R, Taylor P. Use of a remotely steerable "robotic" catheter in a branched endovascular aortic graft. J Vasc Surg 2012;55:223-5.
12. Riga C, Bicknell C, Cheshire N, Hamady M. Initial clinical application of a robotically steerable catheter system in endovascular aneurysm repair. J Endovasc Ther 2009;16:149-53.

13. Bagla S, Smirniotopoulos J, Orlando JC, Piechowiak R. Robotic-assisted versus manual prostatic arterial embolization for benign prostatic hyperplasia: A comparative analysis. Cardiovasc Intervent Radiol 2017;40:360-5.

14. Rolls AE, Riga CV, Bicknell CD, Regan L, Cheshire NJ, Hamady MS, et al. Robot-assisted uterine artery embolization: A first-in-woman safety evaluation of the magellan system. J Vasc Interv Radiol 2014;25:1841-8.

15. Hansen Medical. Magellan Robotic System. Available from: http://www.hansenmedical.com/us/en/vascular/brochures. [Last accessed on 2018 Jan 06].

16. Rao S. Endovascular robotic catheters: An emerging transformative technology in the interventional radiology suite. Radiol Nurs 2016;35:211-7.

17. Riga CV, Bicknell CD, Wallace D, Hamady M, Cheshire N. Robot-assisted antegrade in-situ fenestrated stent grafting. Cardiovasc Intervent Radiol 2009;32:522-4.

18. Riga CV, Cheshire NJ, Hamady MS, Bicknell CD. The role of robotic endovascular catheters in fenestrated stent grafting. J Vasc Surg 2010;51:810-9.

19. Riga CV, Bicknell CD, Rolls A, Cheshire NJ, Hamady MS. Robot-assisted fenestrated endovascular aneurysm repair (FEVAR) using the magellan system. J Vasc Interv Radiol 2013;24:191-6.

20. Kassamali RH, Ladak B. The role of robotics in interventional radiology: Current status. Quant Imaging Med Surg 2015;5:340-3.

21. Bismuth J, Duran C, Stankovic M, Gersak B, Lumsden AB. A first-in-man study of the role of flexible robotics in overcoming navigation challenges in the iliofemoral arteries. J Vasc Surg 2013;57:14S-9S.

22. Khan EM, Frumkin W, Ng GA, Neelagaru S, Abi-Samra FM, Lee J, et al. First experience with a novel robotic remote catheter system: Amigo ${ }^{\mathrm{TM}}$ mapping trial. J Interv Card Electrophysiol 2013;37:121-9.

23. Introduction to CorPath GRX: Next Generation Cardiovascular Robotics. Available from: https://www.dicardiology.com/videos/ introduction-corpath-grx-next-generation-cardiovascular-robotics. [Last accessed on $2018 \mathrm{Apr}$.

24. Weisz G, Metzger DC, Caputo RP, Delgado JA, Marshall JJ, Vetrovec GW, et al. Safety and feasibility of robotic percutaneous coronary intervention: PRECISE (Percutaneous robotically-enhanced coronary intervention) study. J Am Coll Cardiol 2013;61:1596-600.

25. Cercenelli L, Bortolani B, Marcelli E. CathROB: A Highly compact and versatile remote catheter navigation system. Appl Bionics Biomech 2017;2017:2712453.

26. Muller L, Saeed M, Wilson MW, Hetts SW. Remote control catheter navigation: Options for guidance under MRI. J Cardiovasc Magn Reson 2012;14:33.

27. Da Costa A, Guichard JB, Maillard N, Romeyer-Bouchard C, Gerbay A, Isaaz K, et al. Substantial superiority of niobe ES over niobe II system in remote-controlled magnetic pulmonary vein isolation. Int J Cardiol 2017;230:319-23.

28. Riga CV, Bicknell CD, Hamady M, Cheshire N. Tortuous iliac systems - A significant burden to conventional cannulation in the visceral segment: Is there a role for robotic catheter technology? J Vasc Interv Radiol 2012;23:1369-75.

29. Rao S. Robot-assisted transarterial chemoembolization for hepatocellular carcinoma: initial evaluation of safety, feasibility, 
success and outcomes using the Magellan system. Vasc Interv Radiol 2018;26:S12.

30. Riga CV, Bicknell CD, Hamady MS, Cheshire NJ. Evaluation of robotic endovascular catheters for arch vessel cannulation. J Vasc Surg 2011;54:799-809.

31. Bale R, Widmann G. Navigated CT-guided interventions. Minim Invasive Ther Allied Technol 2007;16:196-204.

32. Koethe $\mathrm{Y}, \mathrm{Xu} \mathrm{S}$, Velusamy G, Wood BJ, Venkatesan AM. Accuracy and efficacy of percutaneous biopsy and ablation using robotic assistance under computed tomography guidance: A phantom study. Eur Radiol 2014;24:723-30.

33. Arnolli MM, Hanumara NC, Franken M, Brouwer DM, Broeders IA. An overview of systems for CT- and MRI-guided percutaneous needle placement in the thorax and abdomen. Int $\mathrm{J}$ Med Robot 2015;11:458-75.

34. Won HJ, Kim N, Kim GB, Seo JB, Kim H. Validation of a CT-guided intervention robot for biopsy and radiofrequency ablation: Experimental study with an abdominal phantom. Diagn Interv Radiol 2017;23:233-7.

35. Gianfelice D, Lepanto L, Perreault P, Chartrand-Lefebvre C, Milette PC. Value of CT fluoroscopy for percutaneous biopsy procedures. J Vasc Interv Radiol 2000;11:879-84.

36. Silverman SG, Tuncali K, Adams DF, Nawfel RD, Zou KH, Judy PF, et al. CT fluoroscopy-guided abdominal interventions: Techniques, results, and radiation exposure. Radiology 1999;212:673-81.

37. Kato R, Katada K, Anno H, Suzuki S, Ida Y, Koga S, et al. Radiation dosimetry at CT fluoroscopy: Physician's hand dose and development of needle holders. Radiology 1996;201:576-8.

38. Stoianovici D, Cleary K, Patriciu A, Mazilu D, Stanimir A, Craciunoiu N, et al. AcuBot: A robot for radiological interventions. IEEE Trans Robot Autom 2003;19:927-30.

39. Cleary K, Melzer A, Watson V, Kronreif G, Stoianovici D. Interventional robotic systems: Applications and technology state-of-the-art. Minim Invasive Ther Allied Technol 2006;15:101-13.

40. Cleary K, Stoianovici D, Patriciu A, Mazilu D, Lindisch D, Watson V, et al. Robotically assisted nerve and facet blocks: A cadaveric study. Acad Radiol 2002;9:821-5.

41. Patriciu A, Solomon SB, Kavoussi LR, Stoianovici D. Robotic kidney and spine percutaneous procedures using a new laser-based CT registration method. MICCAI 2001 Lecture Notes in Computer Science. Vol. 2208. 2001. p. 249-57.

42. Solomon SB, Patriciu A, Bohlman ME, Kavoussi LR, Stoianovici D. Robotically driven interventions: A method of using CT fluoroscopy without radiation exposure to the physician. Radiology 2002;225:277-82.

43. Kronreif G, Kettenbach J, Figl M, Kleiser L, Ptacek W, Fürst M. Evaluation of a robotic targeting device for interventional radiology. Int Congr Ser 2004;1268:486-91. Available from: http://www.sciencedirect.com/science/article/pii/ S0531513104007174. [Last accessed on $2018 \mathrm{Apr}$.

44. Kettenbach J, Kronreif G, Figl M, Fürst M, Birkfellner W, Hanel R, et al. Robot-assisted biopsy using computed tomography-guidance: Initial results from in vitro tests. Invest Radiol 2005;40:219-28.

45. Martinez RM, Ptacek W, Schweitzer W, Kronreif G, Fürst M, Thali MJ, et al. CT-guided, minimally invasive, postmortem needle biopsy using the B-rob II needle-positioning robot. J Forensic Sci 2014;59:517-21.

46. Maurin B, Bayle B, Piccin O, Gangloff J, de Mathelin M, Doignon C, et al. A patient-mounted robotic platform for CT-scan guided procedures. IEEE Trans Biomed Eng 2008;55:2417-25.
47. Walsh CJ, Hanumara NC, Slocum AH, Shepard JA, Gupta R. Patient-mounted, telerobotic tool for CT-guided percutaneous interventions. J Med Device 2008;2:11007. Available from: http://www.medicaldevices.asmedigitalcollection.asme.org/ article.aspx?articleid=1473780. [Last accessed on 2018 May].

48. Seitel A, Walsh CJ, Hanumara NC, Shepard JA, Slocum AH, Meinzer HP, et al. Development and evaluation of a new image-based user interface for robot-assisted needle placements with the Robopsy system. Proc SPIE 2009;7261: 72610X-72610X-9. Available from: https://www.proceedings. spiedigitallibrary.org/proceeding.aspx?doi=10.1117/12.811507. [Last accessed on 2018 May].

49. Kettenbach J, Kara L, Toporek G, Fuerst M, Kronreif G. A robotic needle-positioning and guidance system for CT-guided puncture: Ex vivo results. Minim Invasive Ther Allied Technol 2014;23:271-8.

50. Perfint Healthcare Pvt., Ltd. Available from: http://www. perfinthealthcare.com/. [Last accessed on 2018 May].

51. Zhou Y, Thiruvalluvan K, Krzeminski L, Moore WH, Xu Z, Liang Z, et al. CT-guided robotic needle biopsy of lung nodules with respiratory motion - Experimental system and preliminary test. Int J Med Robot 2013;9:317-30.

52. Tovar-Arriaga S, Vargas JE, Ramos JM, Aceves MA, Gorrostieta E, Kalender WA, et al. A fully sensorized cooperative robotic system for surgical interventions. Sensors (Basel) 2012;12:9423-47.

53. Kettenbach J, Kronreif G. Robotic systems for percutaneous needle-guided interventions. Minim Invasive Ther Allied Technol 2015;24:45-53.

54. Zangos S, Melzer A, Eichler K, Sadighi C, Thalhammer A, Bodelle B, et al. MR-compatible assistance system for biopsy in a high-field-strength system: Initial results in patients with suspicious prostate lesions. Radiology 2011;259:903-10.

55. Kettenbach J, Kubin K, Stadler A, Schernthaner M, Lammer JM. Pneumatically driven robotic system for MR-guided biopsie, drainage and tumorablation: first clinical experiences. J Vasc Interv Radiol 2008;19:S60.

56. Yang B, Roys S, Tan UX, Philip M, Richard H, Gullapalli R, et al. Design, development, and evaluation of a master-slave surgical system for breast biopsy under continuous MRI. Int J Rob Res 2014;33:616-30.

57. Park SB, Kim JG, Lim KW, Yoon CH, Kim DJ, Kang HS, et al. A magnetic resonance image-guided breast needle intervention robot system: Overview and design considerations. Int J Comput Assist Radiol Surg 2017;12:1319-31.

58. Johns Hopkins Medicine. MrBot: The First Fully-Actuated MRI Robot. Available from: http://www.urobotics.urology.jhu.edu/ projects/MrBot/. [Last accessed on 2018 May].

59. Stoianovici D, Kim C, Petrisor D, Jun C, Lim S, Ball MW, et al. MR safe robot, FDA clearance, safety and feasibility prostate biopsy clinical trial. IEEE ASME Trans Mechatron 2017;22:115-26.

60. Laboratoire TIMC-IMAG. LPR: A Light Puncture Robot for CT and MRI Interventions. Available from: http://www-timc.imag.fr/ article853.html. [Last accessed on 2018 May].

61. Bricault I, Zemiti N, Jouniaux E, Fouard C, Taillant E, Dorandeu F, et al. Light puncture robot for $\mathrm{CT}$ and MRI interventions: Designing a new robotic architecture to perform abdominal and thoracic punctures. IEEE Eng Med Biol Mag 2008;27:42-50.

62. Monfaredi R, Wilson E, Azizi Koutenaei B, Labrecque B, Leroy $\mathrm{K}$, Goldie $\mathrm{J}$, et al. Robot-assisted ultrasound imaging: Overview and development of a parallel telerobotic system. Minim Invasive Ther Allied Technol 2015;24:54-62. 
63. Boctor EM, Fischer G, Choti MA, Fichtinger G, Taylor RH. A dual-armed robotic system for intraoperative ultrasound guided hepatic ablative therapy: A prospective study. IEEE Int Conf Robot Autom 2004 Proceedings ICRA '04 2004. Vol.3. 2004. p. 2517-22.

64. Liang K, Rogers AJ, Light ED, Von Allmen D, Smith SW. Simulation of autonomous robotic multiple-core biopsy by 3D ultrasound guidance. Ultrason Imaging 2010;32:118-27.

65. Mebarki R, Krupa A, Chaumette F. 2-D ultrasound probe complete guidance by visual servoing using image moments. IEEE Trans Robot 2010;26:296-306.

66. Janvier MA, Durand LG, Cardinal MH, Renaud I, Chayer B, Bigras $\mathrm{P}$, et al. Performance evaluation of a medical robotic 3D-ultrasound imaging system. Med Image Anal 2008;12:275-90.
67. Nouaille L, Vieyres P, Poisson G. Process of optimisation for a 4 DOF tele-echography robot. Robotica 2012;30:1131-45.

68. Vieyres P, Poisson G, Courreges F, Merigeaux O, Arbeille P. The TERESA project: From space research to ground tele-echography. Ind Rob 2003;30:77-82.

69. Arbeille P, Capri A, Ayoub J, Kieffer V, Georgescu M, Poisson $\mathrm{G}$, et al. Use of a robotic arm to perform remote abdominal telesonography. AJR Am J Roentgenol 2007; 188:W317-22.

70. Essomba T, Laribi MA, Gazeau JP, Zeghloul S, Poisson G. Contribution to the design of robotized tele-echography system. Front Mech Eng [Internet]. 2012;7:135-49. Available from: https:/ doi.org/10.1007/s11465-012-0326-3. [Last accessed on 2018 May]. 